chemical properties. As a designation for the whole groups of hormones having a structure reminiscent of the sterols, Callow and Young ${ }^{1}$ suggested the name of 'steroid' which has generally been accepted. The Greek ending 'oid' derived from $\varepsilon \iota \sigma \circ \varsigma$ meaning form, has often been used in pharmacology to designate compounds similar in their actions to those described by the work preceding this ending (for example, toxoid). Accordingly, the four main types of steroid hormone-like actions might be described as corticoid, luteoid, folliculoid and testoid. Thus without introducing any essentially novel terms, we could classify the steroid hormones into four main pharmacological groups according to the principles mentioned above :

Corticoid $=$ having activity of : cortin, adrenal cortical hormone, principle maintaining life of adrenalectomized animals, etc.

Luteoid = having activity of : progestin, corpus luteum hormone, progesterone, luteine, $\beta$-hormone, kythine, luteohormone, corporin, relaxin, mucifying hormone, luteocrinine, etc.

Folliculoid = having activity of : cestrin, œstrogenic hormone, feminine, gynæecogenic hormone, menformon, folliculin, $\alpha$-hormone, follicular hormone, female hormone, etc.

Testoid = having activity of : androkinin, male hormone, testis hormone, androgenic hormone, etc.

It may incidentally be mentioned that the term 'cortin' has now lost its original meaning as a generic term for adrenal cortical hormone activity since Hartman et al. ${ }^{2}$ now distinguish between the "vital factor, cortin" and the "sodium factor". This means that desoxycorticosterone acetate is both a 'cortin' and a 'sodium factor', while some of Hartman's cortin preparations are apparently devoid of the latter activity. If this type of subdivision were carried over to the group of the testoids, androsterone would have to be called prevailingly a 'prostate factor' and testosterone a 'seminal vesicle factor'. Subdivision carried to this degree is of no particular value as it eventually leads to the mere statement of single characteristics which can much better be done by the generally accepted practice of merely stating the degree to which the compound has 'metrotropic', 'mammotropic', 'renotropic', pituitary-enlarging, lifemaintaining, etc., ability. While the determination of all these activities is evidently of importance, it is felt that for the reasons mentioned above, the single action can be used as a basis for a classification. On the other hand, the classification suggested in this note has none of these deficiencies, and the proposed group names are equally applicable to artificial compounds and true hormones. It is hoped they will prove useful at this time when so much work is done on a multitude of newly synthetized steroid compounds with hormone activity.

Department of Anatomy; Hans Selye.

MeGill University,

Montreal.

June 16.

${ }^{1}$ Callow, R. K., and Young, F. G., Proc. Roy. Soc., A, 157, 194 (1936). 'Hartman, Frank A., Spoor, H. J., and Lewis, I. A., Science, 89' 204 (1939).

\section{Nomenclature of Pituitary Autacoids}

Tне nomenclature of pituitary autacoids is unsatisfactory, and current terminology with reference to those of the anterior lobe is particularly at fault. The terms thyrotropic and gonadotropic are in general use. These terms are misleading. The Greek

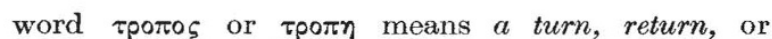
turning about and the verb $\tau \rho \varepsilon \pi \omega$ from the same root can be used transitively to signify direct or guide.

We have long-established precedent for this root in current biological literature. The phototropic or geotropic behaviour of a plant signifies that it turns towards or away from light or the centre of the earth. The same words have also been used to describe the behaviour of an animal when it moves to or from a source of light or the earth's centre. Students of animal behaviour have very properly recognized that this is inconsistent with scientific usage. Consequently they have substituted phototaxis and geotaxis.

Dr. A. S. Parkes has suggested the use of the term gonadotrophic instead of gonadotropic ${ }^{1}$. The meaning of the Greek root of the suffix trophic would indicate that the hormone feeds the gonads. This is far from certain and it is possible to choose a more appropriate root which can be applied to all the activities of the pituitary gland. It is certain that there is some physiological connexion or link between the specific activities of the anterior lobe and the thyroid, gonads, etc.

One Greek word which meets our requirements is $\delta \varepsilon \sigma \mu \circ$, which means a bond, fetter, or link. This suggests the following terminology for the anterior lobe autacoids :

(1) thyrodesmic (thyrotropic)

(2) blastodesmic or growth-promoting

(3) oodesmic or follicle-stimulating

(4) xanthodesmic (luteinizing)

(5) androdesmic or testicle-stimulating desmie

(6) galactodesmic (galactogogue)

One objection to the items of this list is that growth-promoting is sufficiently explicit. For those who prefer native words to international terms, thyroid-stimulating is certainly better than thyrotropic.

Existing terminology of the posterior lobe autacoids is not open to the same objections. The term oxytocie is not inherently inconsistent with established usage of Greek affixes. Still, it is open to the criticism that it contains no familiar root which suggests its meaning. For the English-speaking scientific worker, uterine stimulant is therefore a better term than oxytocic activity. From every point of view Hogben's melanophore stimulant is preferable to chromatropic. Until it is possible to distinguish between the substances responsible for melanophore stimulation and erythrophore stimulation the adjectives melanodesmic and erythrodesmic might be appropriately used. Pressor substance is sufficiently explicit from the point of view of the English-speaking world and is in accordance with international usage. So also is the term anti-diuretic. For those who prefer a uniform nomenclature, I suggest the following :

(1) hæmodesmic (pressor)

(2) splanchnodesmic (oxytocic)

(3) (a) melanodesmic $(b)$ erythrodesmic

(4) nephrodesmic

(5) leucodesmic (the at present hypothetical " $W$ ", substance of. Hogben and Slome).

Natural History Department, F. W. LANDGREBE.

University of Aberdeen. June 23.

${ }^{1}$ NATURE, 141, 36, 73 (1938). 